\title{
DISCLAIMER
}

DCRL --53817

This report was prepared as an account of work sponsored by an agency of the United States Government. Neither the United States Governtuent nor any acepcy thereof, nor any of their employeen, makes any warranty, express or implied, or anumes any legal liability or reaposeibility for the accuracy. completenese, or usefulaces of any itformation, spparatus, product, of process disclowed, or represents that its use would not infringe privately owned rights. Reference herein to any specific commercial product, process, or secvice by trade nums, trademark, manufacturer, or otherwise does not necessarily conatitute or imply its endorsement, recommendation, or favoring by the United States Government of any afeacy thereor. The view and opinions of authors expresed berein do not necesearily state or reflect those of the United States Government or any agency thereor.

\section{A Control System for Maintaining High Stability in Gas Pressure}

\author{
C. R. Wuest \\ C. D. Hendricks
}

Manuscript date: September 1987

\section{LAWRENCE LIVERMORE NATIONAL LABORATORY University of California - Livermore, California - 94550}




\section{Contents}

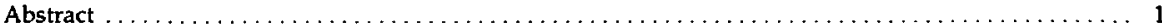

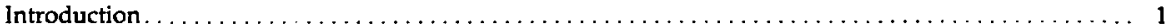

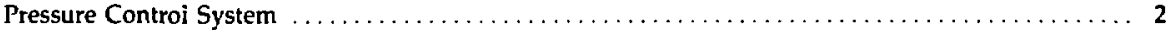

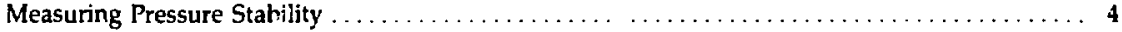

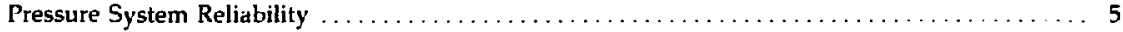

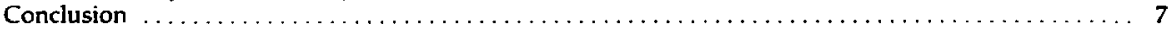

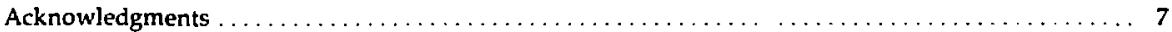

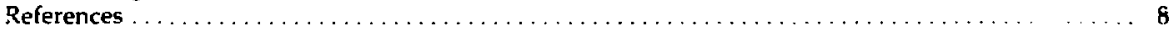




\section{A Control System for Maintaining High Stability in Gas Pressure}

\section{Abstract}

A pressure control system has been implemented on an experiment designed to detect the presence of fractional charges in bulk matter. The experiment utilizes a liquiddroplet generation technique requiring high-stability gas-pressure delivery to ensure accurate data collection. The pressure control system consists of a pressurized mercury reservoir coupled to a reservoir containing a low-vapor-pressure, diffusion-pump oil. A commercially available differential pressure transducer, servo-driven valve, and controller sense the pressure flictuations with respect to a static reference pressure. The system can maintain constant pressure to better than one part in $\mathbf{1 0} 000$ at working pressures in the range of 100 to 300 psi.

\section{Introduction}

A number of control systems have been developed as part of an ongoing experiment at LLNL to detect fractional charge in bulk matter. These systems are necessary to ensure highly accurate data collection. This paper will describe a system designed to minimize pressure variations in a liquid-droplet generator assembly.

We use the well known droplet-generation technique of inducing a Rayleigh breakup of a pressurized jet of liquid emanating from a circular orifice, ${ }^{1}$ a method that generates highly uniform droplets of liquid. The technique relies on the fact that a cylindrical jet of liquid is unstable under the action of surface tension. Any capillary (surface) waves present on the jet will grow exponentially in time and cause the jet to break up into droplets. Our experiment uses the property that a jet perturbed with a periodically varying capillary wave of sufficient amplitude and frequency will break up into droplets with nearly equal mass.

The uniformity of the droplets using this method has been measured to be about one part in 10000 (Ref. 2). The role that pressure plays in this scheme is given in Eqs. (1) and (2). The droplet size is given by ${ }^{3}$ :

$r_{\mathrm{d}}=\left(\frac{3 a^{2} \lambda}{4}\right)^{1 / 3}=\left(\frac{3 \mathcal{C}_{\mathrm{c}} d_{\mathrm{o}}^{2} V_{1}}{f}\right)^{1 / 3}$

where

$r_{\mathrm{d}}=$ droplet radius,

$a=$ jet radius, $\lambda=$ capillary wavelength,

$C_{\mathrm{r}}=$ jet contraction or "necking down" coefficient,

$d_{0}=$ orifice diameter

$V_{\mathrm{i}}=$ jet velocity,

$f$ = capillary wave frequency.

One sees that the radius of a droplet is proportional to the cube root of the velocity or, equivalently, the mass of a droplet is directly proportional to the velocity. In tum, the jet velocity is dependent on a number of parameters, given approximately by:

$V_{1}=\frac{P a^{2}}{8 \mu l}$.

where

$P=$ pressure at the input of the orifice,

$\mu=$ dynamic viscosity of the liquid.

$l$ = length of the orifice.

In deriving Eq. (2), it is assumed that the pressure at the input end of the orifice is much greater than at the output end. Also, there is an effective lower pressure threshold under which a jet will not form, so Eq. (2) tends to overestimate the velocity for a given pressure. For our purposes, Eq. (2) gives the necessary relation that pressure and velocity are directly proportional; thus, from Eq. (1), pressure and droplet mass are directly proportional.

Figure 1 shows a measurement of droplet velocity versus pressure from a $15.8-\mu \mathrm{m}$-diam orifice 


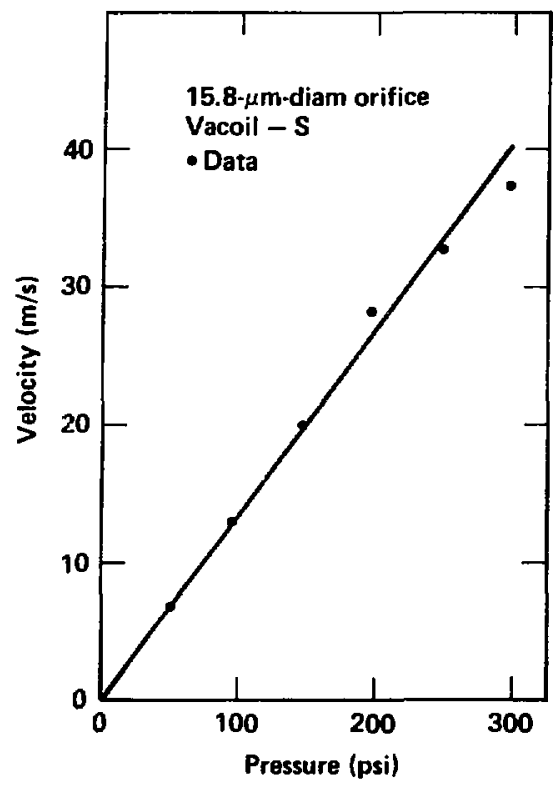

Figure 1. Droplet velocity versus pressure for Vacoil-S from a 15.8- $\mu$ m-diam orifice. The circles represent the data and the line shows the best fit to the data.

for a low-vapor-pressure, low-viscosity, diffusion pump oil produced by the Vacoil Company. The oil has a vapor pressure of $5 \times 10^{-8}$ Torr and a viscosity of $18 \mathrm{cP}$ at $20^{\circ} \mathrm{C}$. The veloxity was determined by measuring the distance between droplets using strobe light illumination. A television camera with magnifying optics and a standard monitor were used to display the droplets. Calibration of the camera image was done by focusing on a micrometer re:icula and marking $100-\mu \mathrm{m}$ steps on the face of the monitor. The measured distance was then multiplied by the known droplet frequency to get the velocity. The error in the velocity is estimated to be about $5 \%$ and is due to uncertainty in the distance measured between droplets. From Fig. 1, it is seen that velocity and pressure are approximately linearly related as expected from Eq. (2).

In order to determine a droplet's electric charge accurately, pressure fluctuations should be kept to a minimum. Any mass variations between droplets will cause droplets of the same charge to be deflected through different distances in our charge spectrometer. Figure 2 shows a computergenerated Monte Carlo simulation of our detector's response for 5000 droplets. The simulation assumes that there is a $1 \%$ variation in longitudinal (jet) velocity due to pressure fluctuations. Also, the droplets are assumed to have zero transverse velocity. From Fig. 2, it can be seen that (for typical conditions in our experiment) pressure changes can cause a smearing of the charge resolution of the order of tens of microns. This smearing, when taken with other sources of noise on the jet (mechanical vibration and small but significant transverse velocity components) can make it difficult, if not impossible, to detect droplets with fractional electric charge.

\section{Pressure Control System}

In response to this problem, a pressure system was developed to minimize fluctuations and give high-stability pressure delivery over periods of hours. The initial system, outlined in Fig. 3, consists of a standard nitrogen gas cylinder with a two-stage regulator that can be set in the range of 0 to 300 psi, with 200 psi being a typical working pressure. A high-pressure reservoir containing mercury at room temperature is pressurized, allowing the mercury to be displaced into another high-pressure reservoir containing the working fluid of the experiment.

The mercury is denser than the oil and thus acts as a frictionless liquid piston, displacing the oil into the droplet generator. $A$ pressure control system (Fig. 4) was built (1' lllow pressurization of the system and to sense the mercury level in each reservoir; it depressurizes the system upon shutdown and also when the mercury level is high in the oil reservoir. At this point, a transfer procedure al!.ows pressurization of the oil reservoir to transfer the mercury back to its reservoir. The oil reservoir is then refilled. The sysiem is fail safe, releasing its pressure in the event of a power failure.

Mercury was chosen to pressurize the oil because it prevents nitrogen gas from being dissolved in the oil. Gas in the liquid oil would cause problems when the droplets are generated in a high vacuum; the escaping gas would impart 


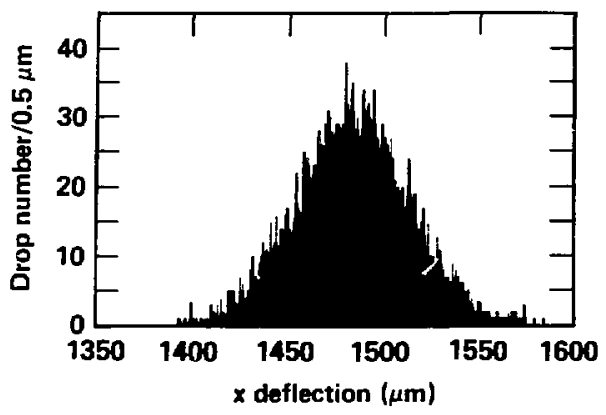

Droplet diameter $=\mathbf{4 0} \mu \mathrm{m}$

Plate voltage $=30 \mathrm{kV}$

Mean z velocity $=30 \mathrm{~m} / \mathrm{s}$

$x$ velocity jitter $=0 \mu \mathrm{s}$

2 velocity jitter $=30 \mathrm{~cm} / \mathrm{s}$

Figure 2. Computer simulation of 5000 dropiets generated in a model detector. The droplets all have a charge of 10 electrons and the longitudinal velocity component is allowed to vary by $1 \%$ with a gaussian distribution around $30 \mathrm{~m} / \mathrm{s}$.

Droplet generator

Pressure system

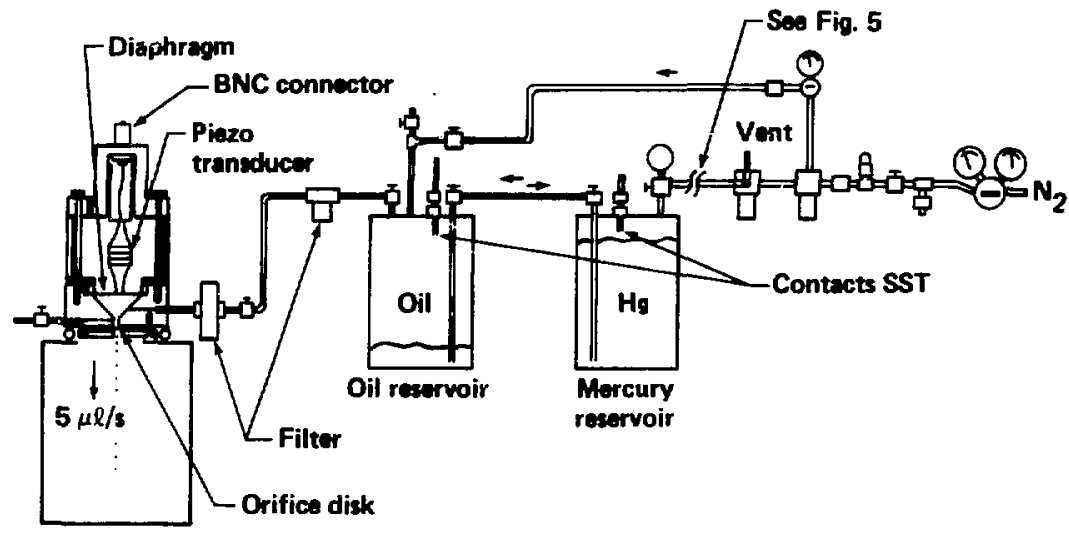

Vecuum chamber

Figure 3. Schematic diagram of the first generation pressure control system for the fractional charge experiment. 


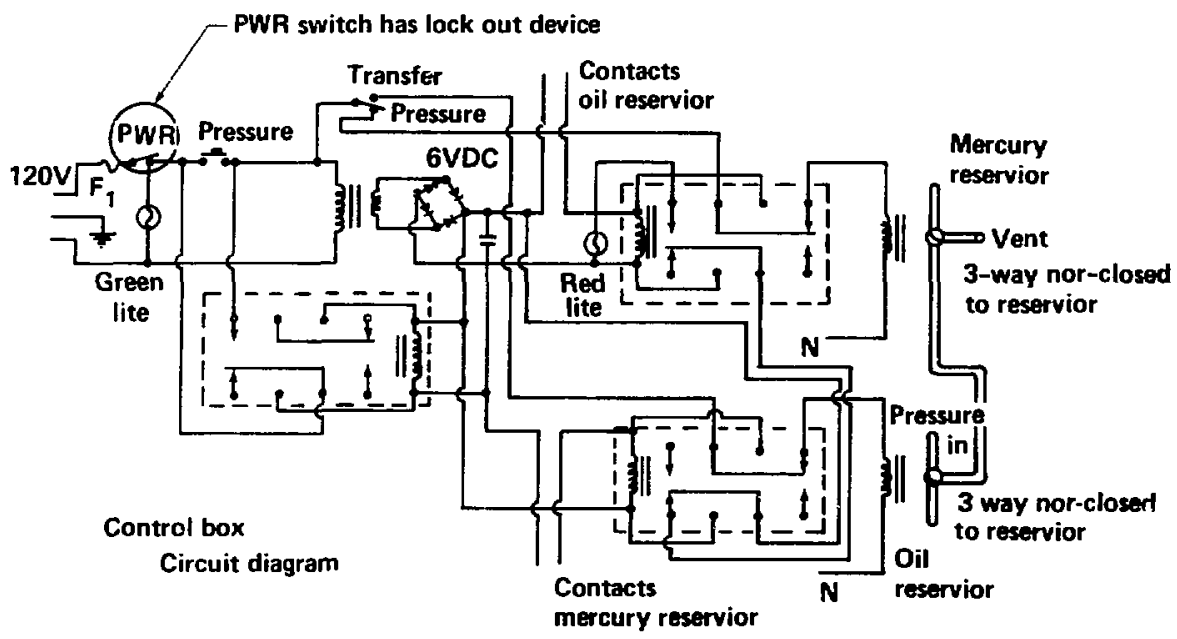

Figure 4. Schematic diagram of the pressure control circuit used in the first generation system.

transverse motion on the droplets, decreasing the charge resolution. Another reason for choosing mercury is that it is relatively inert in the presence of the oil, keeping the oil system free from contamination; the closed system also keeps experimenters safe from the hazards of mercury vapor.

\section{Measuring Pressure Stability}

A study was carried out to measure the stability of the pressure delivered to the system with the droplet generator in operation over periods of hours. The measurement was made with a singleended pressure transducer, demodulator and strip chart recorder provided by the LLNL Pressure Calibration Group. A working pressure of about $100 \mathrm{psi}$ was chosen. Measurements of the pressure as a function of time exhibited a sawtooth behavior with a gradual decrease in pressure of about 0.5 psi over a few hours followed by a rapid (a few seconds) increase in pressure to the nominal set pressure. This behavior is typical of two-stage regulators. The long time behavior is a function of the flow rate out of the droplet generator (typically microliters per second) and the leak rate of the joints in the pressure system.

As a result of the above data, a pr.... back subsystem was developed to achieve a higher degree of stability in pressure delivery to the droplet generator. This subsystem, shown in Fig. 5, is located between the automatic valve pressurizing the mercury reservoir and the nanual input valve on the reservoir (see its platen:ent in Fig. 3). In addition, a metering needle vaive was installed in the line immediately after the twostage regulator. This valve is used to control the rate of gas flow into the system.

The pressure feedback subsystem consists of a differential pressure transducer and feedback control circuit coupled to a servo-driven needle valve in a bleed line. The transducer is the Validyne Corporation's model DP15 with a 0.125 -psi diaphragm installed. The transducer senses pressure differentials on the diaphragm and delivers a voltage proportional to pressure, with the polarity of the voltage indicating positive or negative differential pressure. In this system, \pm 0.125 psi gives an output voltage of $\pm 10 \mathrm{~V}$. An automatic valve is installed in parallel with the transducer and is connected to an over-pressure sensor set to open the valve if the pressure differential is too great. The over-pressure circuit (Fig. 6) senses the absolute value of the transducer voltage and latches the automatic valve open if the voltage rises above a set reference voltage. The valve is closed by pushing a reset on the over-pressure controller. This is necessary to prevent straining the diaphragm beyond its elastic 


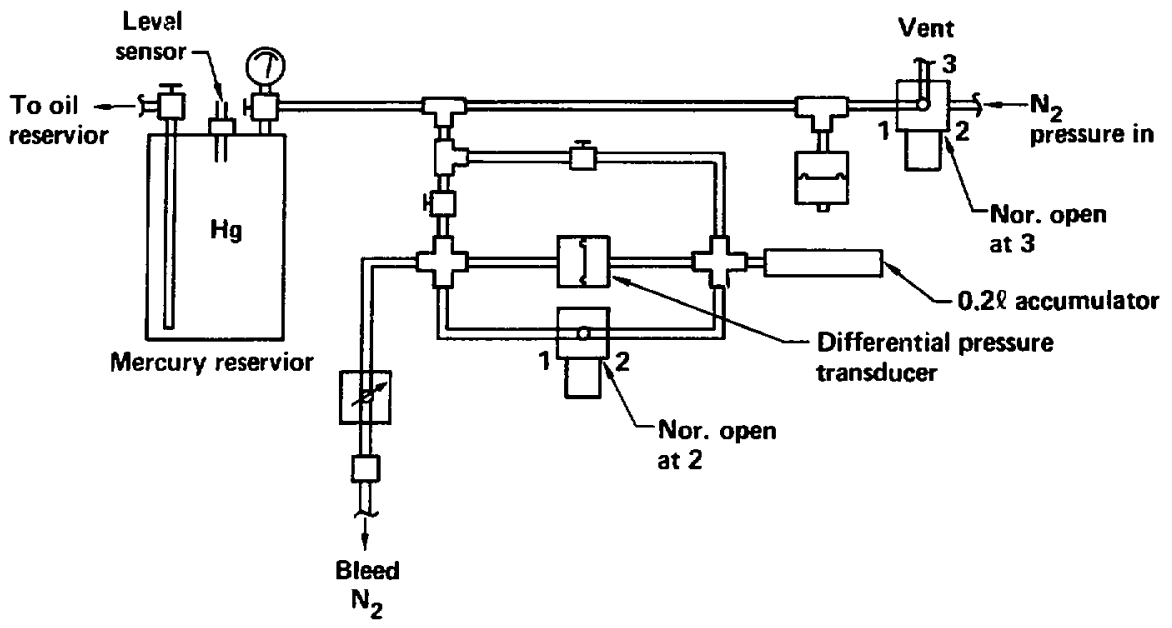

Figure 5. Schematic diagram of the pressure feedback subsystem.

limit. Manual valves are arranged so that a fixed static pressure can be maintained on one side of the diaphragm while dynamic pressure is sensed on the other.

Feedback control of the pressure is achieved by sensing the pressure transducer voltage with the Granville-Phillips Company's model 216020 automatic pressure controller connected to a Granville-Phillips series 216 servo-driven needle valve on a bleed line. The system senses pressure changes occurring at the transducer and opens or closes the servo-driven needle valve accordingly to adjust the amount of gas bled off the pressure feed line. When conditions are right, the system is stable and attempts to maintain pressure such that the voltage out of the transducer is zero. Under these conditions, the working pressure is matched to the pressure on the static side of the diaphragm. Small fluctuations in pressure in this setup can be measured by monitoring the voltage out of the transducer on a strip chart recorder. Figure 7 shows a typical measurement for a working pressure of $170 \mathrm{psi}$. The variations are periodic and correspond to the reset time of the servodriven valve controller. The peak to peak amplitude of each fuctuation is about $1 \mathrm{~V}$ or about 0.0125 psi, giving a stability of better than one part in 10000 .

The error in this measurement can be estimated from the pressure transducer manufactur- er's quoted errors: $0.01 \%$ full-scale thermal zero shift, $1 \%$ full-scale zero shift per $1000 \mathrm{psi}$, and $\pm 0.5 \%$ full scale linearity with $\pm 0.5 \%$ hysteresis. We conservatively estimate the total error to be less than 5\% of actual reading. Most of this error can be attributed to the finite response time of the automatic pressure controller and the servodriven valve.

\section{Pressure System Reliability}

The pressure system has been run for as long as $8 \mathrm{~h}$ with high stability. The system is reasonably straightforward to set up and we have developed a standard procedure for its startup and shutdown. With proper adjustment of the metering valve in series with the two-stage regulator, the system is stable. We have found that if this valve is opened too much, the system can become unstable or exhibit large oscillations as it attempts to regulate the pressure. Also, we have found that by setting the two-stage regulator about 5 psi higher than the desired working pressure on the static side of the pressure transducer, stable operation is achieved quickly (typically, less than $5 \mathrm{~min}$ ) and a minimum of nitrogen is wasted out of the bleed line. It is worth pointing out that this system is inherently insensitive to temperature since both sides of the transducer diaphragm are at the same temperature and the gas-flow rates are small. 


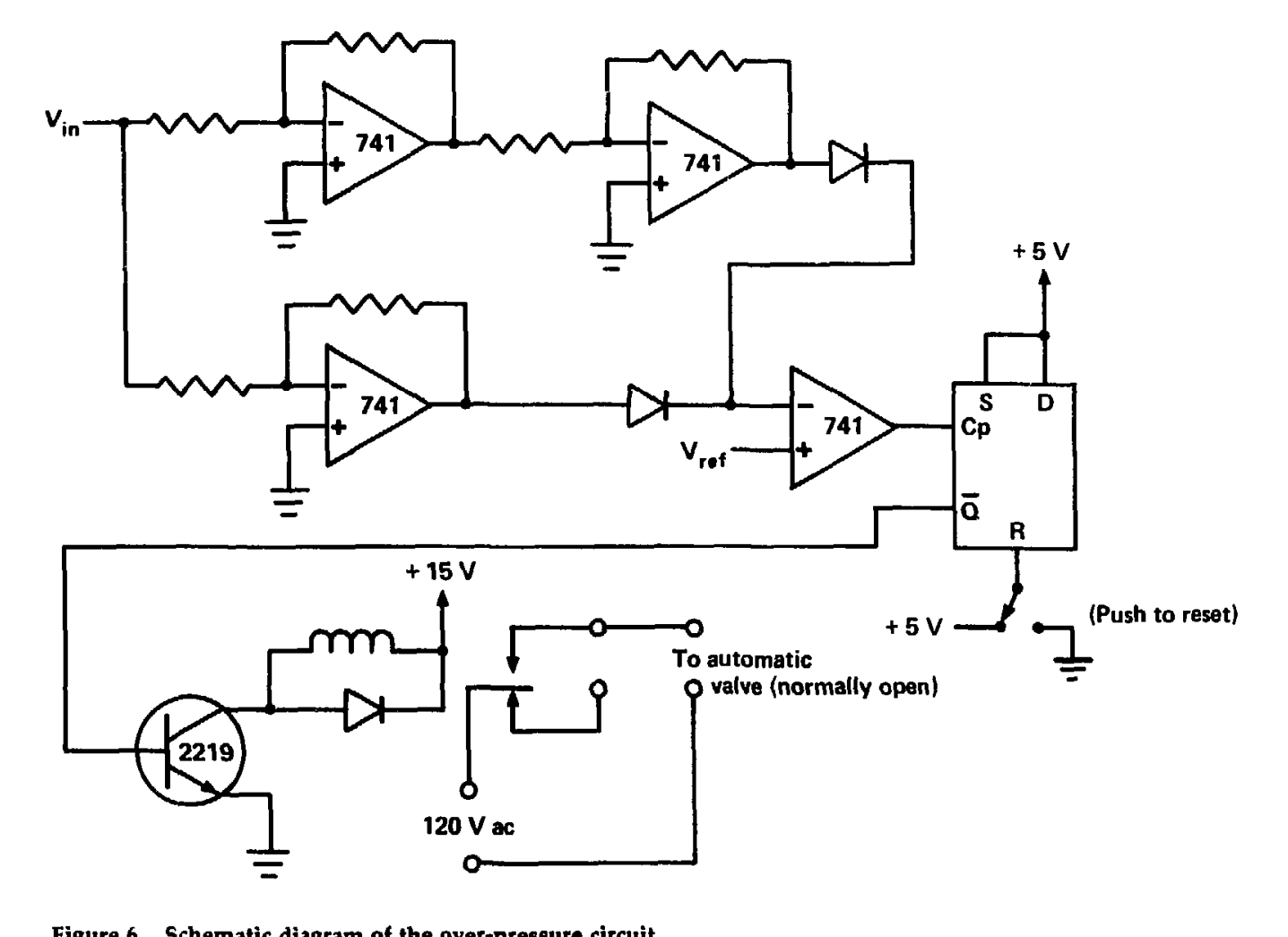




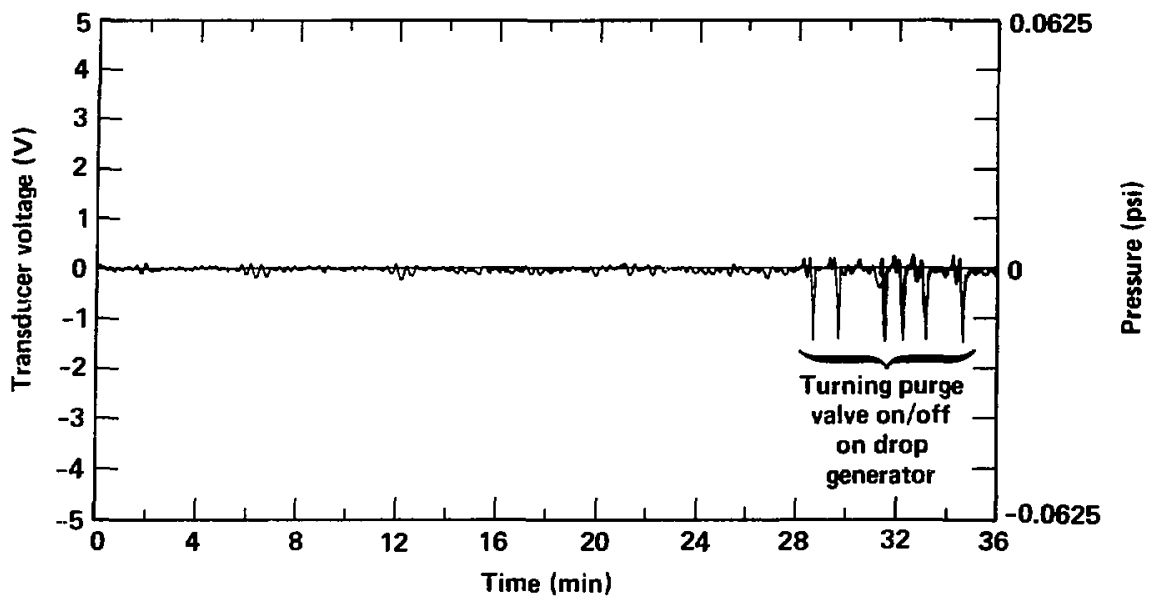

Figure 7. A portion of data showing pressure versus time for the complete pressure control system with feedback.

\section{Conclusion}

In conclusion, pressure regulation using commercially available pressure sensing and control apparatus, together with custom built pressuredelivery, flow-control, and over-pressure systems, has been shown to be capable of achieving pressure stability to better than one part in 10000 . Care must be taken to control gas-flow rates into the feedback loop to prevent unstable operation. In particular, a needle valve inserted in the gas supply line to restrict the flow has been found to be necessary for stable operation. Recent tests have shown that an accumulator stabilizes the feedback circuit further. With proper adjustments of the flow restricting needle valve and the feedback gain and phase delay controls on the GranvillePhillips controller, stability on the order of one part in $10^{6}$ has been observed.

There are diaphragms available for the DP15 series transducers that allow sensing of pressure in the range $\pm 0.08 \mathrm{psi}$. In theory, this diaphragm could be used to achieve another order of magnitude in stability. We believe that great care would have to be taken in the arrangement of the valves in this case, namely, opening and closing the valves would have to be done slowly to prevent minute pressure surges that could damage the diaphragm. However, the basic method of control described in this paper is still applicable in this case.

\section{Acknowledgments}

We would like to recognize the contributions made by R. Lochner, E. Pierce and R. Poli. Also, the LLNL Pressure Calibration group has provided support in the form of technical expertise and hardware. 


\section{References}

1. Lord Rayleigh, Proc. Lond. Math. Soc. 10, 4-13 (1879).

2. G. Hirsch, R. Hagstrom, and C. Hendricks, A Sensitive Method for Detecting Stable Fractional Charges on Matter, Lawrence Berkeley Laboratory, Berkeley, Calif., Report No. 9350 (June 1979).

3. J. M. Schneider and C. D. Hendricks, "Source of Uniform-Sized Liquid Droplets," Rev. Sci. Instr. 35, 1349-1350 (1964); N. R. Lindblad and J. M. Schneider, "Production of Uniform-Sized Liquid Droplets," I. Sci. Instrum. 42, 635-638 (1965).

$\mathrm{MLR} / \mathrm{mp}$ 